\title{
Um método de amostragem para fluxos instáveis
}

\author{
Paul Krause \\ Universidade Federal de Santa Catarina - Departamento de Matemática \\ 88040-900, Campus Trindade, Florianópolis, SC \\ E-mail: p.krause@ufsc.br \\ Pedro L. S. Dias \\ Universidade de São Paulo - Departamento de Ciências Atmosféricas \\ 05508-090, Cidade Universitária, São Paulo, SP \\ E-mail: pldsdias@lncc.br
}

\begin{abstract}
Resumo: Apresenta-se um novo método, ISMC, de re-amostragem do estado de um sistema de equações diferenciais ordinárias que possua variáveis determinantes no sentido de que, quando estas variáveis são controladas com valores extraídos de uma solução do sistema, a dinâmica das variáveis restantes, ou marginais, é contrativa. Através de perturbações, o ISMC acomoda mais perto da realidade os valores amostrais de variáveis marginais usando valores de controle para variáveis determinantes. $O$ método é testado com as equações de Lorenz (1963) e um sistema obtido por perturbação destas com funções seno. Os resultados apontam para um aumento da previsibilidade de sistemas instáveis.
\end{abstract}

\section{Introdução}

Um grande obstáculo à previsão precisa é a instabilidade dinâmica [13]. Instabilidade está na natureza da maioria dos processos não-lineares de retorno ao equilíbrio de sistemas ambientais como a água e o ar, cujas dinâmicas são geralmente complexas [4]. Em tais situações, o método de Monte Carlo (MC) com amostragem baixa pode sofrer de falta de robustez nas estimativas que produz [8] e com amostragem alta pode sofrer de excesso de confiança, já que neste caso apenas os eventos de maior probabilidade são recolhidos nos intervalos de confiança. Neste trabalho, a ser publicado em [11], um novo método de re-amostragem do estado de um sistema de equações diferenciais ordinárias (e.d.o.), chamado de Influence Sampling - Monte Carlo (ISMC), é apresentado para sistemas possuindo um conjunto de variáveis, ditas determinantes [5,9], que se continuamente controladas com valores de uma trajetória do sistema fazem com que as demais variáveis, ditas marginais, tenham uma função de fluxo contrativa [1,6]. O ISMC é um método de controle discreto de variáveis determinantes de tais sistemas. Ele perturba os valores amostrais de variáveis marginais usando valores de controle para variáveis determinantes. Diferente dos métodos Bred Vector [10] e Singular Vector [2], adotados nos centros de previsão de tempo, o ISMC perturba valores amostrais do estado a fim de acomodar estes valores mais próximo dos valores reais e não para amostrar espaços instáveis da dinâmica local. Neste trabalho, o ISMC é testado em "modo previsão" com as equações de Lorenz [12], L63, e o sistema L63+ obtido por perturbação das equações de Lorenz com termos de fonte (funções seno). Este último tem uma dinâmica instável modulada pelos termos de fonte, como é comumente o caso nos modelos de previsão de tempo. 


\section{Método e Resultados}

Considera-se um sistema de e.d.o não-linear, autônomo, $\mathrm{dx} / \mathrm{dt}=\mathrm{f}(\mathrm{x}), \mathrm{f} \in \mathrm{C}^{1}\left(\mathrm{R}^{\mathrm{n}}\right)$, com trajetórias em um compacto do $R^{n}$, e escreve-se o problema a valor inicial $d x / d t=f(x), t \geq t_{0}, x\left(t_{0}\right)=x_{0}$, na forma $\mathrm{d} \Phi_{\mathrm{t}} / \mathrm{dt}=\mathrm{L} \Phi_{\mathrm{t}}, \Phi_{\mathrm{t} 0}\left(\mathrm{x}_{0}\right)=\mathrm{x}_{0}$, onde $\Phi_{\mathrm{t}}(\mathrm{x})$ é a função de fluxo do sistema de e.d.o. e $\mathrm{L}=\mathrm{f}\left(\mathrm{x}_{0}\right) \cdot \nabla$. Após uma série de transformações adicionais do problema a valor inicial, aplica-se a fórmula $\mathrm{e}^{(\mathrm{t}-\mathrm{t} 0)(\mathrm{A}+\mathrm{B})} \mathrm{u}\left(\mathrm{x}_{0}\right)=\mathrm{e}^{(\mathrm{t}-\mathrm{t} 0) \mathrm{A}} \mathrm{u}\left(\mathrm{x}_{0}\right)+\int_{\mathrm{t} 0}^{\mathrm{t}} \mathrm{e}^{(\mathrm{t}-\mathrm{s}) \mathrm{L}} \mathrm{B}{ }^{(\mathrm{s}-\mathrm{t} 0) \mathrm{A}} \mathrm{u}\left(\mathrm{x}_{0}\right)$ ds, de decomposição da ação $\mathrm{e}^{(\mathrm{t}-\mathrm{t}) \mathrm{L}}$, com $\mathrm{A}=\mathrm{PL}, \mathrm{B}=\mathrm{QL}$ e $\mathrm{u}\left(\mathrm{x}_{0}\right)=\mathrm{PL} \mathrm{x}$, onde $\mathrm{PL}=\mathrm{f}\left(\mathrm{x}_{0}, \mathrm{y}_{0},{ }^{\prime}\right) \cdot \nabla$ e QL=L-PL, onde, ainda, (x', $\left.x^{\prime}\right)$ é uma partição do estado x em dois grupos de variáveis e $y_{0}$ " um valor fixo de $x "$, para chegar-se a $\left.\Phi_{\mathrm{t}}{ }^{\prime}\left(\mathrm{x}_{0}{ }^{\prime}, \mathrm{x}_{0}{ }^{\prime \prime}\right) \approx \Phi_{\mathrm{t}}{ }^{\prime} \mathrm{x}_{0}{ }^{\prime}+\mathrm{dx}_{0}{ }^{\prime}(\mathrm{t}), \mathrm{y}_{0}{ }^{\prime \prime}\right)$ com $\mathrm{dx}_{0}{ }^{\prime}(\mathrm{t})=\left(\Phi_{\mathrm{t}}{ }^{\prime}\left(\mathrm{x}_{0}{ }^{\prime}, \mathrm{x}_{0}{ }^{\prime \prime}\right)-\mathrm{x}_{0}{ }^{\prime}\right)-\int{ }_{\mathrm{t} 0}^{\mathrm{t}} \mathrm{f}^{\prime}\left(\Phi_{\mathrm{s}}{ }^{\prime}\left(\mathrm{x}_{0}{ }^{\prime}, \mathrm{x}_{0}{ }^{\prime \prime}\right), \mathrm{y}_{0}{ }^{\prime \prime}\right) \mathrm{ds}$.

Este resultado permite deduzir o método ISMC de re-amostragem do estado de um sistema de e.d.o. por perturbação dos valores amostrais de variáveis marginais do sistema [11]. O método vale para sistemas não-autônomos e tem custo comparável ao de Monte Carlo se aplicadas perturbações esparsas. Os resultados, a seguir, provêm de teses conduzidos sobre o ISMC em "modo previsão" com o L63 e L63+.
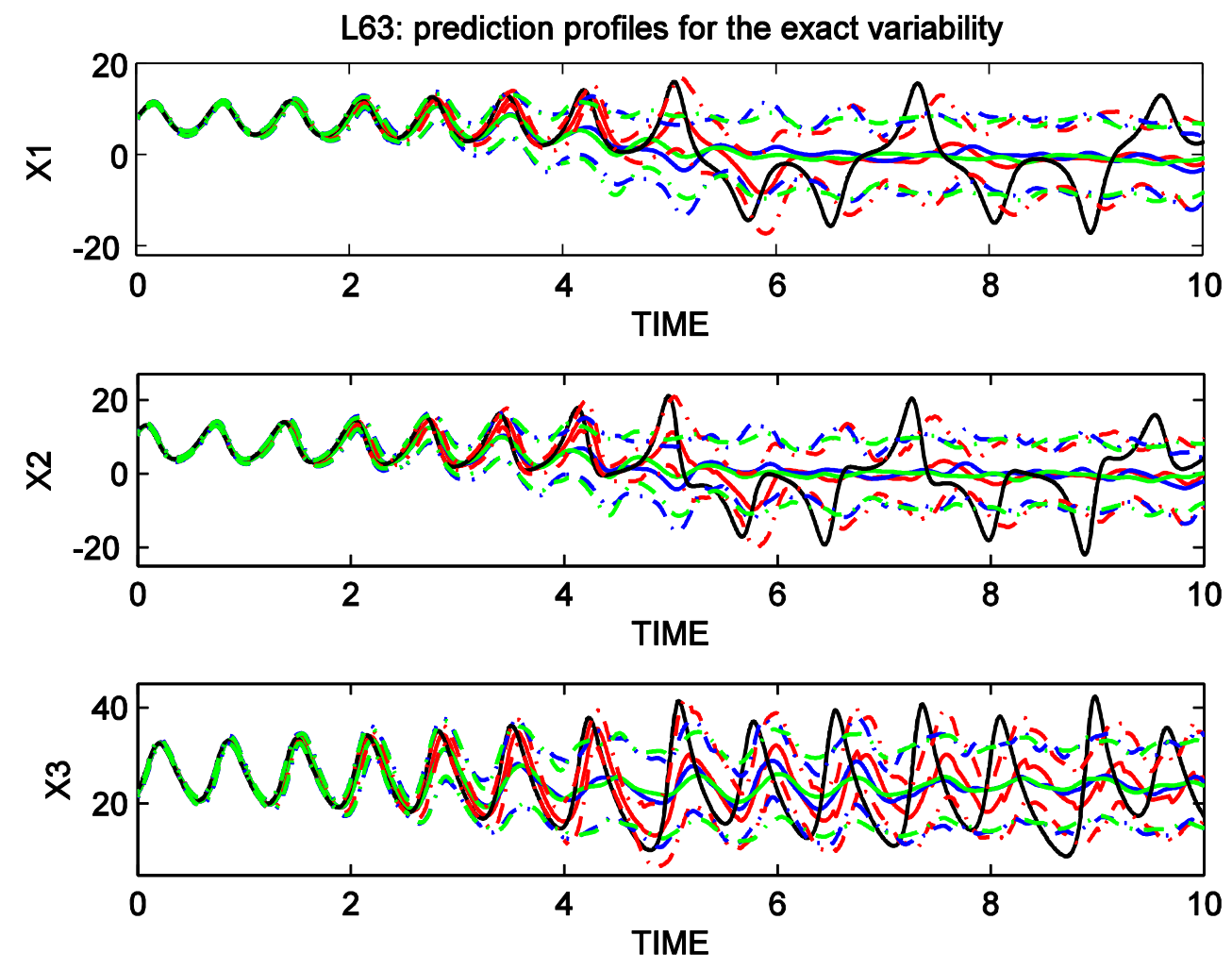

Figura 1: Previsão (média amostral +/- desvio padrão) da variabilidade exata de um evento do L63: ISMC/50 (vermelho), MC/50 (azul) e MC/200 (verde). 
L63: prediction error profiles for the exact variability
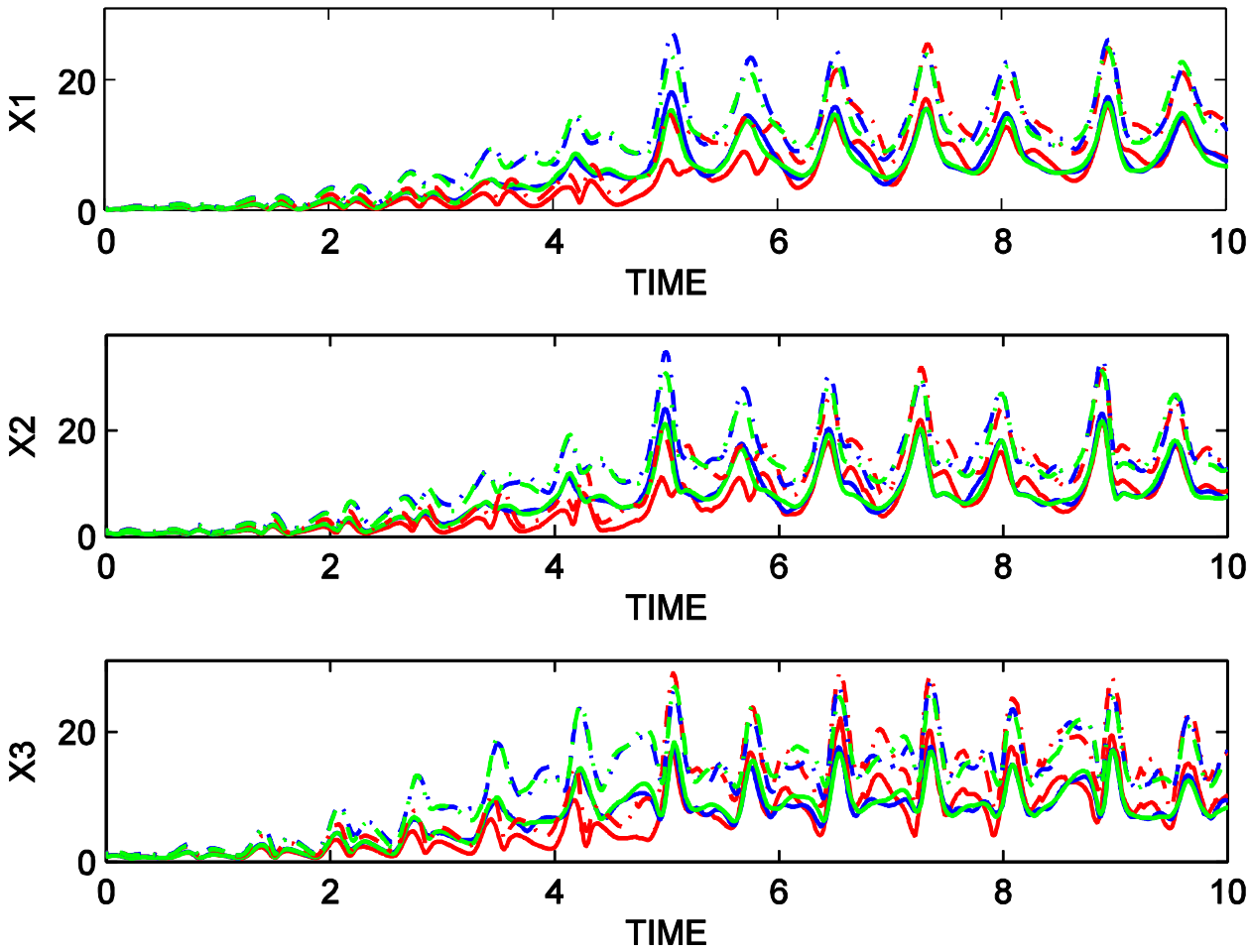

Figura 2: Erro da previsão da variabilidade exata de um evento do L63: ISMC/50 (vermelho), $\mathrm{MC} / 50$ (azul) e MC/200 (verde).
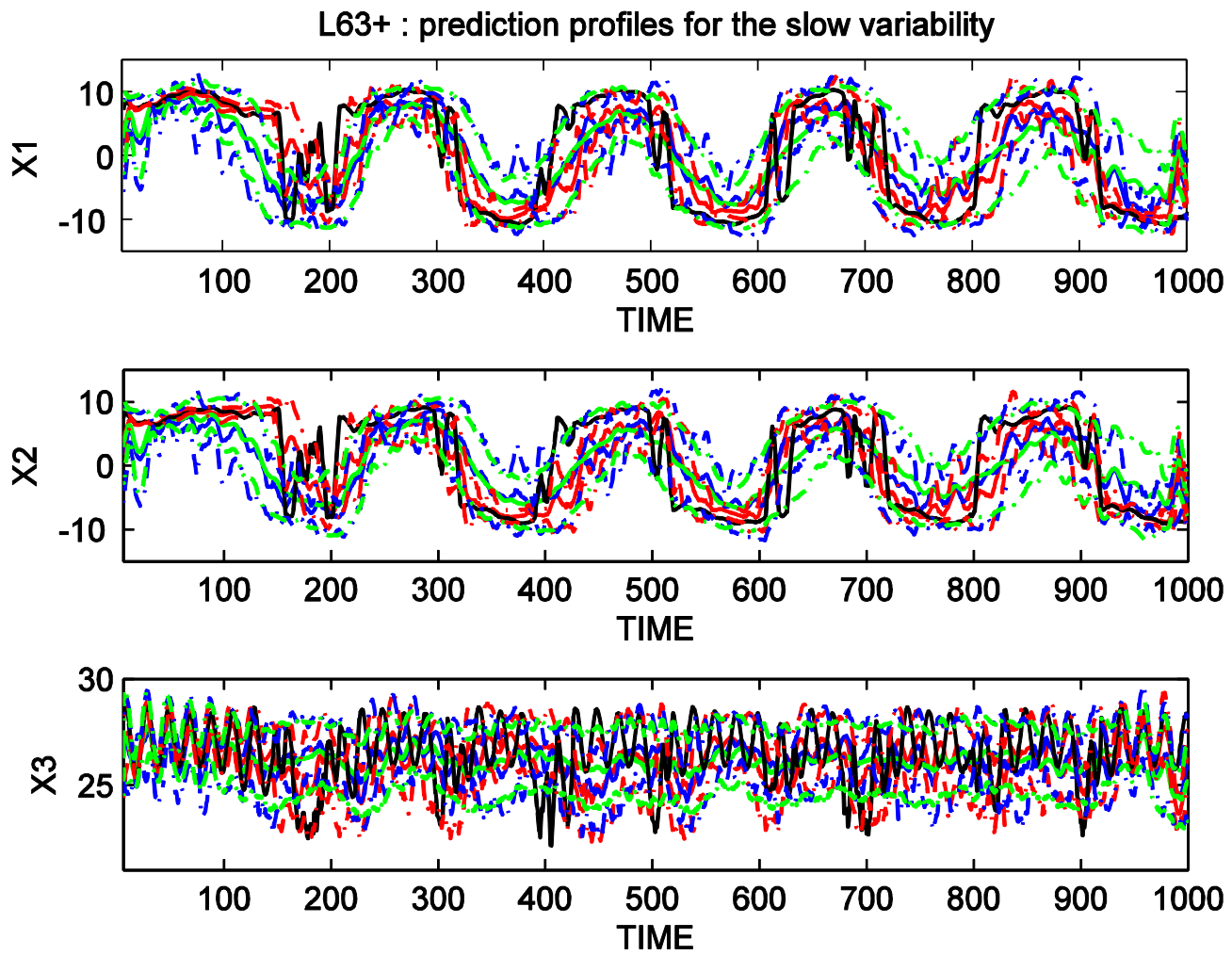

Figura 3: Previsão (média amostral +/- desvio padrão) da variabilidade lenta (média temporal das componentes do estado) de um evento do L63+: ISMC/10 (vermelho), MC/10 (azul) e MC/200 (verde). 

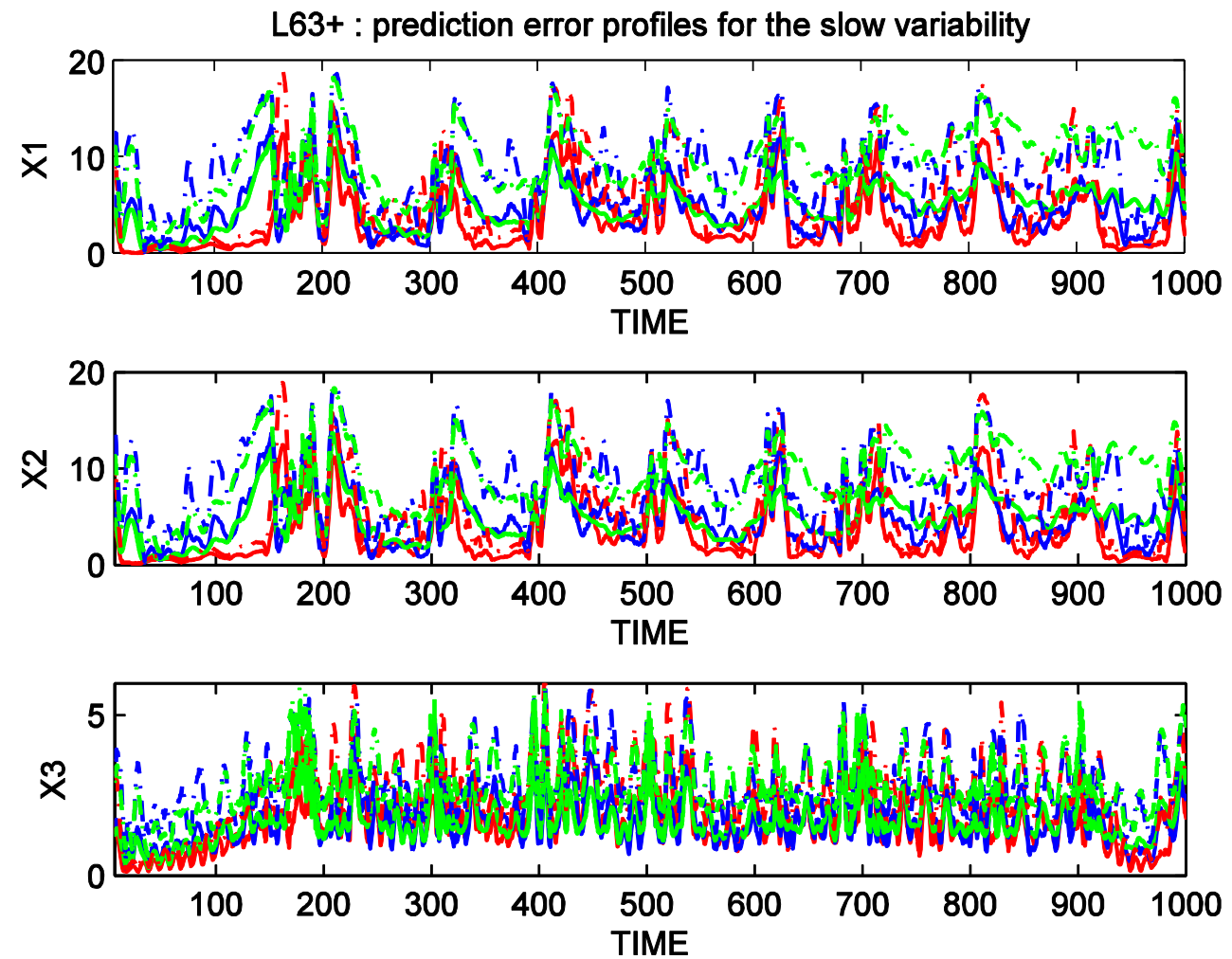

Figura 4: Erro da previsão da variabilidade lenta de um evento do L63+: ISMC/10 (vermelho), MC/10 (azul) e MC/200 (verde).

\section{Conclusão}

Apresentou-se um novo método, ISMC, de re-amostragem do estado de um sistema de equações diferenciais ordinárias (e.d.o.) que possua variáveis determinantes no sentido de que, quando estas variáveis são controladas com valores extraídos de uma solução do sistema, a dinâmica das variáveis restantes, ou marginais, é contrativa. Através de perturbações, o ISMC acomoda mais perto da realidade os valores amostrais de variáveis marginais usando valores de controle para variáveis determinantes. O método tem custo comparável ao de Monte Carlo se aplicadas perturbações esparsas. Sobre ele foram conduzidos testes com as equações de Lorenz, L63, e o sistema L63+ obtido por perturbação das equações de Lorenz com funções seno. Para ambos os sistemas, a variável $\mathrm{x} 1$ mostrou-se determinante em experimentos numéricos. Os testes de previsão foram feitos tomando-se a média amostral de $\mathrm{x} 1$ como valor de controle para esta variável. Foram analisadas as previsibilidades forte e fraca de dois eventos instáveis, ou seja, duas soluções instáveis dos sistemas. Um evento é dito fortemente previsível em determinado intervalo de tempo se uma previsão forte, ou seja, de sua variabilidade, pode ser feita com margem de confiança estreita ao longo do intervalo em questão. Um evento é dito fracamente previsível em determinado instante se uma previsão fraca (ou estatística), ou seja, do estado no instante em questão, pode ser feita com margem de confiança estreita naquele instante. Nos testes, o ISMC mostrou-se capaz de aumentar a previsibilidade de ambas as soluções instáveis dos sistemas L63 e L63+. 


\section{Referências}

[1] S. Banach, Sur les opérations dans les ensembles abstraits et leur application aux équations intégrales, Fund. Math., 3 (1922) 133-181.

[2] R. Buizza, "The singular vector approach to the analysis of perturbation growth in the atmosphere", Tese de Doutorado, University of London, 1997.

[3] A. J. Chorin, O. H. Hald, R. Kupferman, Optimal prediction with memory, Physica D, 166 (2002) 239-257.

[4] J.-P. Eckmann, D. Ruelle, Ergodic theory of chaos and strange attractors, Rev. Mod. Phys., 57:3(I) (1985) 617-656.

[5] C. Foias, G. Prodi, Sur le comportement global des solutions non-stationnaires des équations de Navier-Stokes en dimension 2, Rend. Sem. Mat. Univ. Padova, 39 (1967) 1-34.

[6] J. A. Goldstein, "Semigroups of Linear Operators and Applications", Oxford University Press, 1985.

[7] G. H. Golub, J. M. Ortega, "Scientific Computing and Differential Equations", Academic Press, 1992.

[8] H. Kahn, A. W. Marshall, Methods of reducing sample size in Monte Carlo computations, Operations Research, 1 (1953) 263-278.

[9] V. K. Kalantarov, E. S. Titi, Global attractors and determining modes for the 3D NavierStokes-Voight equations, Chin. Ann. Math., 30B(6) (2009) 697-714.

[10] E. Kalnay, Z. Toth, Ensemble forecasting at NMC: the generation of perturbations, Bull. Amer. Met. Soc., 74:12 (1993) 2317-2330.

[11] P. Krause, P. L. S. Dias, On the influence sampling of unstable flows, Nonlinearity, submetido

[12] E. N. Lorenz, Deterministic nonperiodic flow, J. Atm. Sci., 20 (1963) 130-141.

[13] A. J. Majda, Challenges in climate science and contemporary applied mathematics, Comm. Pure App. Math., LXV (2012) 920-948. 